\title{
Effect of Rice stripe virus NS3 on Transient Gene Expression and Transgene Co-Silencing
}

\author{
Seong-Han Sohn ${ }^{1 *}$, Sun Mi Huh ${ }^{1}$, Kook-Hyung Kim ${ }^{2}$, Jin Woo Park ${ }^{1}$ and George Lomonossoff ${ }^{\mathbf{3}}$ \\ ${ }^{I}$ National Academy of Agricultural Science, Rural Development Administration, Suwon 441-707, Korea \\ ${ }^{2}$ Department of Agricultural Biotechnology, Seoul National University, Seoul 151-741, Korea \\ ${ }^{3}$ Department of Biological Chemistry, John Innes Centre, Norwich Research Park, Norwich NR4 7UH, UK \\ (Received on July 19, 2011; Accepted on August 30, 2011)
}

\begin{abstract}
Nonstructural protein 3 (NS3) encoded by RNA3 of Rice stripe virus (RSV), known to be a suppressor of gene silencing, was cloned and sequenced. The cloned NS3 gene is composed of 636 nucleotides encoding 211 deduced amino acids, and showed a high degree of similarity with the equivalent genes isolated from Korea, Japan and China. The NS3 gene promoted the enhancement of transient gene expression and suppressed transgene co-silencing. In the transient GFP expression via agroinfiltration, GFP expression was dramatically enhanced in terms of both protein yield and expression period in the presence of NS3. The highest accumulation of GFP protein reached to $6.8 \%$ of total soluble proteins, which corresponded to a two-fold increase compared to that obtained in the absence of NS3. In addition, NS3 significantly suppressed the initiation of GFP co-silencing induced by the additive GFP infiltration in GFP-transgenic Nicotiana benthamiana. The NS3 gene was also found to be a stronger suppressor than Cucumber mosaic virus $2 \mathrm{~b}$. These observations are believed to be derived from the strong suppressive effect of NS3 on gene silencing, and indicate that NS3 could be used as an effective enhancer for the rapid production of foreign proteins in plants.
\end{abstract}

Keywords : agroinfiltration, green fluorescent protein, nonstructural protein 3 (NS3), Rice striped virus, suppressor

Plants are an attractive host system for producing foreign proteins such as biopharmaceuticals; however, the cost and low protein yield are significant problems limiting the commercialization in plant-based molecular farming (Doran, 2006). Foreign proteins can be produced quickly by use of agroinfiltration (Fischer et al., 1999). In this process, Agrobacterium meditates incorporation of T-DNA into plant genome, and the expression of foreign genes on $\mathrm{T}$ -

\footnotetext{
*Corresponding author.

Phone) +82-31-299-1701, FAX) +82-31-299-1707

E-mail)sohnseonghan@korea.kr
}

DNA usually reaches a maximum at 2-3 days post-infiltration (dpi). However, the expression level then declines rapidly due to the activation of post-transcriptional gene silencing (PTGS) (Johansen and Carrington, 2001; Voinnet et al., 2003). To suppress PTGS, viral suppressors such as p25 of Potato virus X (PVX), 2b of Cucumber mosaic virus (CMV), helper component-proteinase (Hc-Pro) of Potato virus $Y$ (PVY) and p19 from Tomato bushy stunt virus (TBSV) have been deployed. Specifically, the p19 of TBSV effectively suppresses the onset of PTGS, and allows stronger and more persistent expression of foreign genes than the other suppressors such as p25 of PVX and p19 of PVY (Vionnet et al., 2003). On the other hand, Hc-Pro increased the protein expression of GFP by more than threefold via agroinfiltration in Nicotiana benthamiana (Ma et al., 2009) and extended the expression period by threefold even using DNA bombardment (Chiera et al., 2008). Recently, use of a hypertranslatable (HT) leader sequence derived from 5'-UTR of Cowpea mosaic virus (CPMV) RNA2 in conjunction with p19 enhanced protein accumulation to levels corresponding to $20-25 \%$ of total extractable protein (Sainsbury and Lomonossoff, 2008). Another result in conjugation with p19 was also reported for GFP production with a variety of regulatory sequences involved in protein synthesis, such as a translational enhancer (TE), a secretory signal peptide (SP), the hydrophobin (HFBI) gene and ER retention signal (KDEL), accumulated to up to $51 \%$ of total soluble protein (TSP) (Joensuu et al., 2010). These large-scale protein productions were believed to be resulted from the suppression of gene silencing by $\mathrm{p} 19$ against the ectopic gene expression in plants. The suppression mechanism by 19 occurs via the direct binding of small RNAs, leading to interference with the PTGS pathway (Lakatos et al., 2006; Mérai et al., 2005). Thus, transient expression systems which include with suppressors can be a powerful tool for the production of large amounts of functional proteins in a short time. The NS3 gene has been identified as a gene silencing suppressor not only in plants and cultured insect cell (Hemmes et al., 
2007), but also in mammalian cells (Schnettler et al., 2008), through the binding and sequestering of siRNAs (Xiong et al., 2009). Here, we isolated the NS3 gene from a Korean isolate and reported its effect on both transient gene expression and transgene co-silencing in comparison with CMV $2 b$ (Choi et al., 2008). As a result, we have demonstrated that the NS3 gene can be one of the most potent enhancers for transient expression and suppressors of gene silencing.

\section{Materials and Methods}

Isolation of NS3 gene and cloning in binary vectors. RSV-infected rice was kindly provided by the Crop Protection Division of National Academy of Agricultural Science (NAAS, Korea). The RSV NS3 gene was amplified from total RNA of RSV-infected rice by RT-PCR using the primers (5'-TTTACTATACAATACACTTCCGAC-3',5'CTAGAGTCCACTAGACAGCCAGTG-3'). The RT-PCR reaction was carried according to the protocol of AccuPower ${ }^{\mathrm{TM}}$ RT/PCR PreMix (Bioneer, Korea). The amplified cDNA was cloned into GEM $^{\circledR}$-T Easy (Promega, USA) and sequenced. The NS3 gene was inserted into the multicloning site of SpeI/EcoRI-digested pGreen0229 to place it under the control of $35 \mathrm{~S}$ promoter to give construct pGreen0229-35S:NS3 (35S-NS3). The 35S-NS3 was transferred into A. tumefaciens strain AGL1, with helper plasmid (pJIC) by electroporation (McCormac et al., 1998).

Agroinfiltration. Agroinfiltration was carried out by using a previously reported protocol (Choi et al., 2008). Wildtype $N$. benthamiana was used to examine the enhancing effect of NS3 on transient expression, and two lines of GFP-transgenic N.benthamiana were used for analyzing the suppressive effect on GFP-transgene co-silencing. The properties of NS3 were compared to those of $2 b$ using the pGreen0229-35S:2b (35S-2b) vector. In addition, pGreen0229-35S:GFP (35S-GFP) was used as a marker vector for transient expression and a initiator of GFPtransgene co-silencing. These two vectors were previously constructed by Choi et al. (2008). GFP-transgenic plants were produced using leaf-disc transformation with $A$. tumefaciens harboring 35S-GFP (Sohn et al., 2011). The expression patterns resulting from agroinfiltration were photographed with an Olympus C-5050Z digital camera under UV illumination (BIB-150P, Spectronics Corp. USA).

Northern blot. Total RNA was extracted using the Trizol ${ }^{\circledR}$ reagent (Invitrogen, USA). Ten micrograms of the RNA was fractionated by electrophoresis on $1.2 \%$ agaroseformaldehyde denaturing gels, and subsequently transferred onto Hybond ${ }^{\mathrm{TM}} \mathrm{N}^{+}$nylon membrane (GE Healthcare, UK).
Hybridization was conducted in Rapid-hyb ${ }^{\mathrm{TM}}$ buffer (GE Healthcare) by the manufacturer's instruction. Probes specific to the GFP, $2 b$ and NS3 genes were labeled with ${ }^{32}$ P-dCTP (Perkin-Elmer, USA) by the random prime labeling system (Rediprime ${ }^{\mathrm{TM}}$ II, GE Healthcare). To visualize the bands, the membrane was exposed to a Phosphor imaging screen (Kodak, USA) for 2 to 16 hours and then analyzed by the Molecular Imager FX system (Bio-Rad, USA).

Western blot and GFP quantifying. For western blotting, total soluble protein (TSP) was extracted from the agroinfiltrated leaves as described by Choi et al. (2008). Protein extracts $(5 \mu \mathrm{g})$ were separated by $12.5 \%$ SDS-PAGE, and subsequently blotted onto polyvinylidene fluoride (PVDF) membranes (GE Healthcare). The GFP protein was detected by incubating with anti-GFP polyclonal antibody (Clontech, USA) followed by incubation with horseradish peroxidase-conjugated anti-rabbit second antibody (GE Healthcare). The peroxdiase reaction was detected by the chemiluminescence method with Super Signal West Dura (Pierce, USA). The accumulated GFP protein content was determined based on the regression line drawn between the densitometric values of GFP standards.

\section{Results and Discussion}

Structure of NS3 gene. The cloned NS3 gene was composed of 636 nucleotides and 211 deduced amino acids. The nucleotide sequence generally shared a high degree of identity, ranging from 97 to $99 \%$, with other NS3 genes already isolated from Korea (Lian et al., 2011), Japan and China (Jonson et al., 2009; Liang et al., 2005). The most similar nucleotide sequence already registered with GenBank, which differs in only two nucleotides, was Accession Y11095 from an isolate from Japan (Fig. 1A). In terms of amino acid sequence, an identical protein (Accession FN650803, unpublished) was found; however, five locations in the nucleotide sequences were different between the two sequences. Other NS3 proteins had identities in the range of 94 to $99 \%$ (Fig. 1B). When nucleotide and amino acid sequence similarities were taken together, the most closely related strain was YG-JN (Accession number FJ602686.1) isolated in Korea. The cloned NS3 gene was named as YGJN1 and inserted into pGreen0229 to construct pGreen0229-35S:NS3 (35S-NS3) as the mentioned in Materials and Methods.

Enhancement of transient gene expression. To examine the possible enhancing effect of NS3 on transient gene expression, Agrobacterium mixtures of GFP alone, GFP $+2 b$ and GFP+NS3 were infiltrated into the different locations 
A

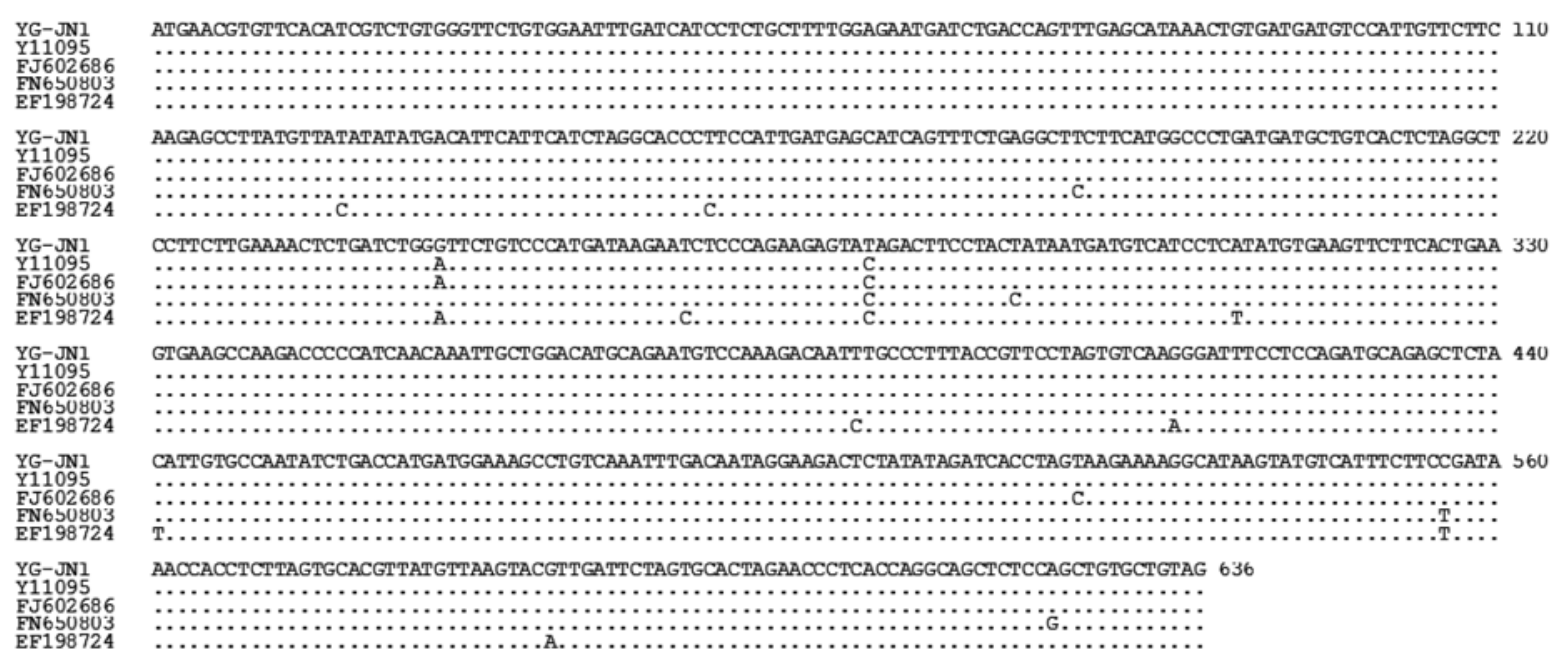

B

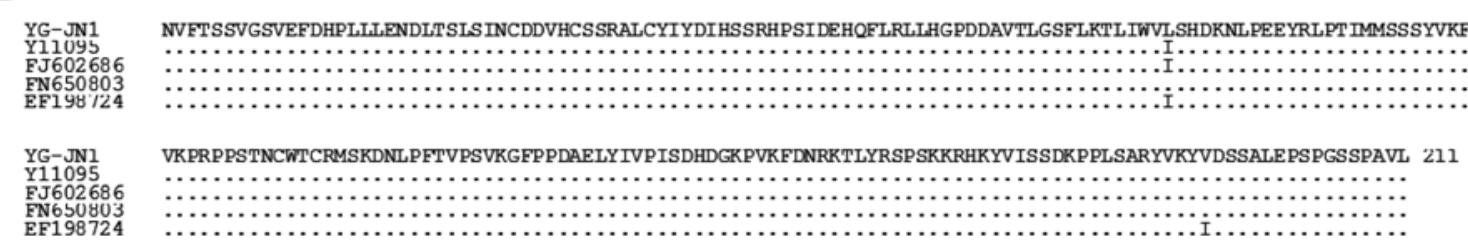

Fig. 1. The multiple alignments of nucleotide (A) and deduced amino acid (B) sequences among NS3 genes. The open reading frame (ORF) consists of 636 nucleotides and 211 amino acids. The NS3 genes compared with YG-JN1 were selected from GenBank (NCBI) only with a high degree of similarity such as Y11095 (Liang et al., 2005), FJ602686 (Jonson et al., 2009), FN650803 (unpublished) and EF198724 (Xiong et al., 2009).

on the same leaf of wild-type N. benthamiana (Fig. 2A). The final concentrations of each Agrobacterium in the mixture was adjusted to $\mathrm{OD}_{600 \mathrm{~nm}}=0.2$ to minimize possible stress on leaf and maximize GFP expression as reported (Choi et al., 2008). The strength of GFP expression was found in the order of GFP+NS3 $>$ GFP $+2 b>$ GFP alone; GFP expression remained until $15 \mathrm{dpi}$ in the presence of NS3 (Fig. 2B). In each case, the level of GFP mRNA peaked at 3 dpi and thereafter gradually declined with the passage of days (Fig. 2C and D). In the presence of the suppressors, the transcripts of GFP RNAs were detected at a significantly higher level and for longer than when GFP was infiltrated alone; significant transcript levels could be detected until 6 or 9 dpi in GFP+2b or GFP+NS3, respectively. These enhanced and extended RNA levels were consistent with NS3 and $2 \mathrm{~b}$ acting as suppressors. The accumulation of GFP was proportional to its RNA levels (Fig. 2F), with the maximum yield of protein reaching 68 ng per $1 \mu \mathrm{g}$ TSP in the presence of NS3 at 6 dpi (Fig. 3). This corresponds to $6.8 \%$ of TSP and represents a more than two-fold compared to that $(2.7 \%)$ achieved with GFP alone. This high-level is similar to that achieved previously using p19 (Meristem Therapeutics, personal communication by Vionnet et al., 2003). On the other hand, the $2 \mathrm{~b}$ protein only increased GFP expression at a maximum of one and half times (4.5\%) compared with GFP alone. This demonstrates that NS3 is more potent than $2 b$ in enhancing transient gene expression. Here, although the concentration of each Agrobacterium $\left(\mathrm{OD}_{600 \mathrm{~nm}}=0.2\right)$ was used to minimize the stress on leaves, concentrations higher than than $\mathrm{OD}_{600 \mathrm{~mm}}$ $=0.2$ could be used to increase the accumulation of GFP further. Therefore, the development of NS3-based transient gene expression could be an excellent system, as it enables the rapid and high-level production of functional proteins, such as vaccines against new strains of infectious disease.

In fact, a transgenic plant-based production of a foreign protein is low and often less than 1\% of TSP (Sheludko, 2008). To overcome this low productivity, the chloroplast transformation technology was introduced and constantly progressed. Although its productivity is relatively higher (5-40\%) (Wang et al., 2009), N- or O-glycosylation process to stabilize and functionalize the proteins is lacking in chloroplasts (Faye and Daniell, 2006). However, recently in conjunction with viral suppressors, breakthrough technologies of transient expressions allowed to produce recombinant proteins up to $50 \%$ of TSP (Joensuu et al., 2010; 
A

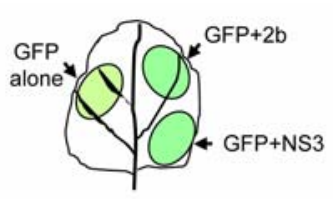

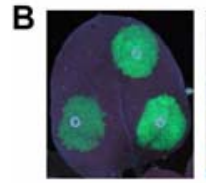

3 dpi

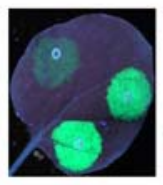

6 dpi

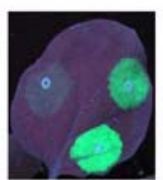

9 dpi

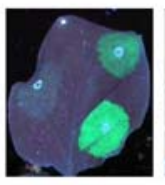

12 dpi

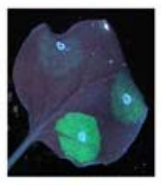

15 dpi

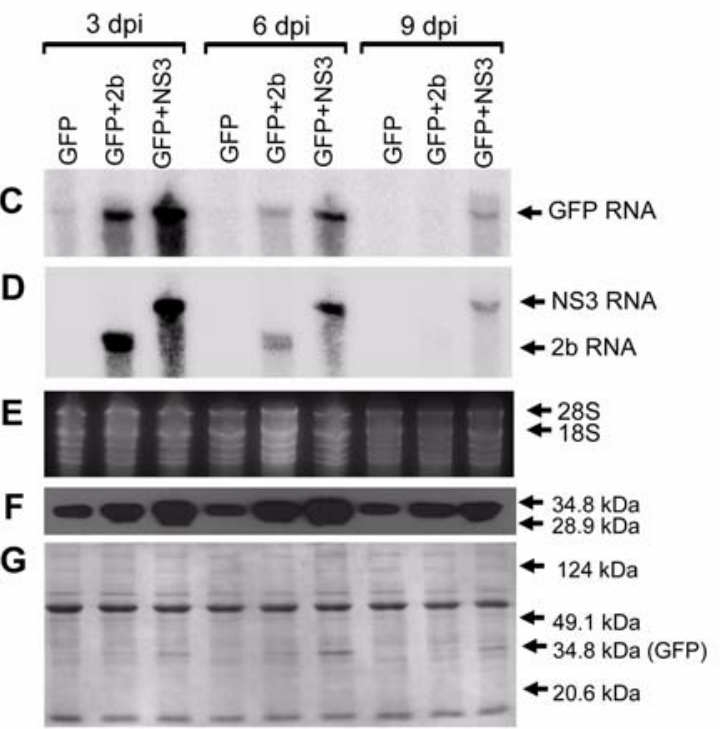

Fig. 2. Effect of NS3 on the transient gene expression via agroinfiltration into wild-type $N$. benthamiana. The Agrobacterium mixtures were infiltrated into three locations on the leaves (A), and the change of GFP expression on the same leaf was tracked until 15 dpi (B). The steady-state RNA levels of GFP (C), 2b (D) and NS3 (D), and the GFP protein accumulation (F) were analyzed. The E and $\mathrm{G}$ indicate the RNA and protein electrophoresed in agarose and SDS-PAGE gels, respectively. GFP alone, Agrobacterium containing 35-GFP; GFP $+2 \mathrm{~b}$, Agrobacterium mixture of 35SGFP and 35S-2b; GFP+NS3, Agrobacterium mixture of 35S-GFP and 35S-NS3.

A

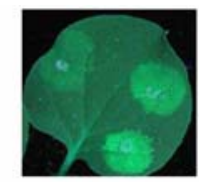

B

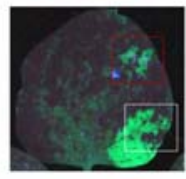

3 dpi
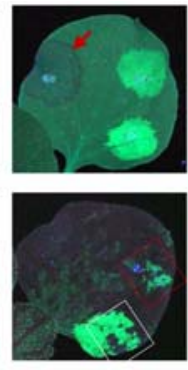

6 dpi
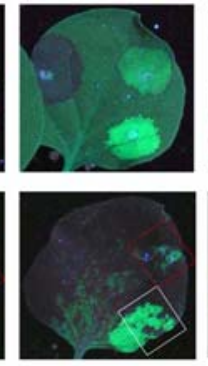

9 dpi

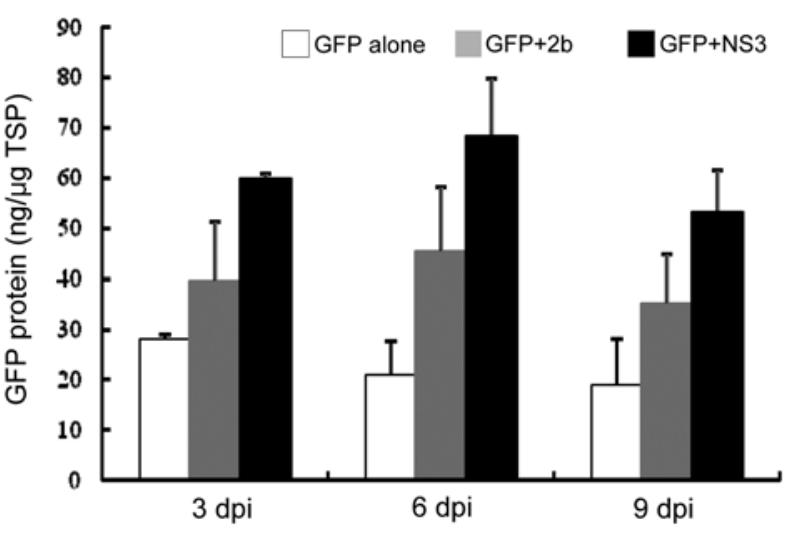

Fig. 3. The accumulation of GFP protein in the absence and presence of $2 b$ or NS3 at 3, 6 and 9 dpi. Accumulation of GFP was determined by western blot-based densitometric analysis and the standard deviations are presented on the bars.

Sanisbury and Lomonossoff, 2008). This demonstrates that effectively-concerted transient expression enables to lead high-level accumulation of useful protein in a short period as much as the chloroplast transformation.

Effect on transgene co-silencing. To examine the suppressive activity of NS3 protein on gene silencing, the Agrobacterium mixtures shown in Fig. 2A were infiltrated into the leaves of GFP-transgenic plants showing different phenotypes such as the steady-green and variegated phenotypes (Fig. 4, Sohn et al., 2011). In plants showing the steady-green phenotype, infiltration of GFP alone initially resulted in GFP expression in addition to that from the transgene-derived GFP. However, at 6 dpi signs of GFP silencing began to appear at the circled-edge of infiltrated zone (red-colored arrow in Fig. 4A). Subsequently, the silencing rapidly co-silenced the transgenic GFP ( 9 to 15 dpi in Fig. 4A). However, in the presence of $2 b$ or NS3, the initiation of silencing was delayed until 12 dpi in the region infiltrated with GFP $+2 b$ or was not observed in that

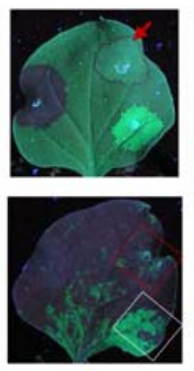

$12 \mathrm{dpi}$

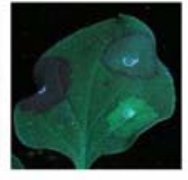

Steady-green

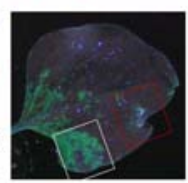

Varigated

$15 \mathrm{dpi}$

Fig. 4. Effect of NS3 on transgene co-silencing in GFP transgenic N.benthamiana. The transgenic plants used here were the steady-green and variegated according to GFP-phenotyping, which respectively represented the uniformed expression in a whole plant and specific silencing in vein regions (Sohn et al., 2011). The locations and mixtures of agroinfiltration were the same as Fig. 2A. The red-colored arrows indicate the initiation of GFP transgene co-silencing. The red and white-lined boxes indicate the regions to track the change of GFP expression over the passage of days. 
infiltrated with GFP+NS3 until 15 dpi. Thus both $2 b$ and NS3 significantly delayed co-silencing of the transgenic GFP, with NS3 being the stronger suppressor. To examine the potential ability of $2 b$ and NS3 to restore transgenic GFP expression in the silencing-established regions, agroinfiltration was conducted using leaves displaying the variegated phenotype (Fig. 4B). As shown in the red and whitelined boxes (Fig. 4B), neither 2b nor NS3 induced changes in the predetermined patterns of GFP expression, which represented the expression topography of transgenic GFP prior to agroinfiltration. However, NS3 successfully suppressed the co-silencing of GFP until $15 \mathrm{dpi}$, while $2 \mathrm{~b}$ lost its suppressive effect at $12 \mathrm{dpi}$ and co-silencing proceeded thereafter. Thus, NS3 is not only the stronger enhancer for transient gene expression but also a stronger suppressor of gene silencing than $2 \mathrm{~b}$.

\section{Acknowledgement}

This study was funded by the National Academy of Agricultural Science (NAAS, PJ006437), Rural Development Administration (RDA), the Republic of Korea in coorperation with John Innes Centre (JIC, Norwich, UK). The JIC is grant-aided by the Biotechnology and Biological Sciences Research Council (BBSRC), UK.

\section{References}

Chiera, J. M., Lindbo, J. A. and Finer, J. J. 2008. Quantification and extension of transient GFP expression by the co-introduction of a suppressor of silencing. Transgenic Res. 17:11431154.

Choi, M. S., Yoon, I.-S., Rhee, Y., Choi, S. K., Lim, S.-H., Won, S.Y., Lee, Y.-H., Choi, H.-S., Lee, S.-C., Kim, K.-H., Lomonossoff, G. and Sohn, S.-H. 2008. The effect of Cucumber mosaic virus $2 \mathrm{~b}$ protein to transient expression and transgene silencing mediated by agro-infiltration. Plant Pathol. J. 24:296-304.

Doran, P. M. 2006. Foreign protein degradation and instability in plants and plant tissue cultures. Trends Biotechnol. 24:426432.

Faye, L. and Daniell, H. 2006. Novel pathways for glycoprotein import into chloroplasts. Plant Biotechnol. J. 4:275-279.

Fischer, R., Vaquero-Martin, C., Sack, M., Drossard, J., Emans, N. and Crmmandeur, U. 1999. Towards molecular farming in the future: transient protein expression in plants. Biotechnol. Appl. Biochem. 30:113-116.

Hemmes, H., Lakatos, L., Goldbach, R., Burgyan, J. and Prins, M. 2007. The NS3 protein of Rice hoja blanca tenuivirus suppresses RNA silencing in plant and insect hosts by efficiently binding both siRNAs and miRNAs. RNA 13:1-11.

Johansen, L. K. and Carrington, J. C. 2001. Silencing on the spot. Induction and suppression of RNA silencing in the Agrobacterium-mediated transient expression system. Plant Physiol. 126:930-938.
Jonson, M. G., Choi, H.-S., Kim, J.-S., Choi, I.-R. and Kim, K.-H. 2009. Complete genome sequence of the RNAs 3 and 4 segments of Rice stripe virus isolates in Korea and their phylogenetic relationships with Japan and China isolates. Plant Pathol. J. 25:142-150.

Joensuu, J. J., Conley, A. J., Lienemann, M., Brandle, J. E., Linder, M. B. and Menassa R. 2010. Hydrophobin fusions for high-level transient protein expression and purification in Nicotiana benthamiana. Plant Physiol. 152:622-633.

Lakatos, L., Csorba, T., Pantoaleo, V., Champman, E. J., Carrington, J. C., Liu, Y.-P., Dolja, V. V., Calvino, L. F., LópexMoya, J. J. and Burgyán, J. 2006. Small RNA binding is a common strategy to suppress RNA silencing by several viral suppressors. EMBO J. 80:5747-5756.

Lian, S., Jonson, M. G., Cho, W. K., Choi, H.-S., Je, Y.-H. and Kim, K.-H. 2011. Generation of antibodies against Rice stripe virus proteins based on recombinant proteins and synthetic polypeptides. Plant Pathol. J. 27:37-43.

Liang, D., Qu, Z., Ma, X. and Hull, R. 2005. Detection and localization of Rice stripe virus gene products in vivo. Virus Genes 31:211-221.

Ma, P., Liu, J., He, H., Yang, M., Li, M., Zhu, X. and Wang, X. 2009. A viral suppressor P1/HC-Pro increases the GFP gene expression in Agrobacterium-mediated transient assay. Appl. Biochem. Biotechnol. 158:243-252.

McCormac, A. C., Elliott, M. C. and Chen, D. F. 1998. A simple method for the production of highly competent cells of Agrobacterium for transformation via electroporation. Mol. Biotech. 9:155-159.

Mérai, Z., Kerényi, Z., Molnár, A., Barta, E., Válóczi, A., Bisztray, G., Havelda, Z., Burgyán, J. and Shihavy, D. 2005. Aureusvirus P14 is an efficient RNA silencing suppressor that binds double-stranded RNAs without size specificity. J. Virol. 79:7217-7226.

Sainsbury, F. and Lomonossoff, G. P. 2008. Extremely high-level and rapid transient protein production in plants without the use of viral replication. Plant Physiol. 148:1212-1218.

Sheludko, Y. V. 2008. Agrobacterium-mediated transient expression as an approach to production of recombinant proteins in plants. Recent Patents Biotech. 2:198-208.

Schnettler, E., Hemmes, H., Goldbach, R. and Prins, M. 2008. The NS3 protein of Rice hoja blanca virus suppresses RNA silencing in mammalian cells. J. Gen. Virol. 89:336-340.

Sohn, S.-H., Choi, M. S., Kim, K.-H. and Lomonossoff, G. 2011. The epigenetic phenotypes in transgenic Nicotiana benthamiana for CaMV 35S-GFP are mediated by spontaneous transgene silencing. Plant Biotech. Rep. 5:273-281.

Voinnet, O., Rivas, S., Mestre, P. and Baulcombe, D. C. 2003. An enhanced transient expression system in plants based on suppression of gene silencing by the 19 protein of Tomato bushy stunt virus. Plant J. 33:949-956.

Wang, H.-H., Yin, W.-B. and Hu, Z.-M. 2009. Advances in chloroplast engineering. J. Genet. Genomics 36:387-398.

Xiong, R., Wu, J., Zhou, Y. and Zhou, X. 2009. Characterization and subcellular localization of an RNA silencing suppressor encoded by Rice stripe tenuivirus. Virology 387:29-40. 